\title{
Close pairs: keys to comprehension of star cluster evolution
}

\author{
Dany Vanbeveren \\ Astrophysical Institute, Vrije Universiteit Brussel, Belgium \\ email: dvbevere@vub.ac.be \\ GroepT Leuven Engineering College, Association KU Leuven, Belgium \\ email: dany.vanbeveren@groept.be
}

\begin{abstract}
In this review I first summarize why binaries are key objects in the study of stellar populations, to understand the evolution of star clusters and galaxies, and thus to understand the universe. I then focus on four specific topics:

(i) the formation (through binaries) and evolution of very massive stars in dense clusters and the importance of stellar-wind mass loss. I discuss preliminary computations of wind mass-loss rates of very massive stars performed with the Munich hydrodynamical code and the influence of these new rates on the possible formation of an intermediate-mass black hole in the cluster MGG 11 in M82;
\end{abstract}

(ii) the evolution of intermediate-mass binaries in a starburst with special emphasis on the variation of the supernova (SN) Ia rate (i.e., on the delayed time distribution of SNe Ia). A comparison with SN Ia rates in elliptical galaxies may provide important clues to SN Ia models as well as to the evolution of SN Ia progenitors;

(iii) the evolution of double-neutron-star mergers in a starburst (i.e., the delayed time distribution of these mergers) and what this tells us about the suggestion that these mergers may be important production sites of r-process elements;

(iv) the possible effect of massive binaries on the self-enrichment of globular clusters.

Keywords. stellar dynamics, binaries: close, stars: evolution

\section{Introduction}

The question of whether or not binaries are important in population studies in general and cluster studies in particular is obviously rhetoric but sometimes it is useful to summarize why this is so. In section 2 , I give a personal selection of observational and theoretical facts that illustrate the importance of binaries. In sections $3-5$, I will highlight three topics related to binaries in clusters/starbursts,

1. one of the most spectacular events related to cluster stellar dynamics is a physical collision of two or more stars. The most probable scenario for this collision process is as follows. A primordial binary or a binary that is formed dynamically interacts with a third object (a single star or another binary). This may result in the formation of unconventionally formed objects (UFOs; Vanbeveren 2007), where binary components are exchanged or where a new binary originates with a component which is a merger of two or more stars. Some standard massive binaries such as the Wolf-Rayet (WR) binary $\gamma^{2}$ Velorum may have been formed this way (see also Vanbeveren 2007). When the interaction results in a merger of two or more stars, the product becomes more massive and attracts other stars. This may initiate a runaway collision process, which in turn may result in the formation of an intermediate-mass black hole. The latter will be discussed in Section 3; 
2. the delay-time distribution of SNe Ia in elliptical galaxies (considered to be remnants of super starbursts) and what this tells us about the binary formation mechanism of these SNe (Section 4);

3. the delay-time distribution of merging double-neutron-star binaries (NS+NS) or merging neutron-star and black-hole binaries $(\mathrm{NS}+\mathrm{BH})$ and the link with the formation of r-process elements (Section 5).

The present population of low-mass stars in globular clusters (GCs) shows clearly the effects of chemical enrichment of a population of intermediate-mass and/or massive stars that formed at an earlier evolutionary phase during the GC's life (see various contributions in these proceedings). Studies aiming at explaining this self-enrichment have mainly focused on single stars. In Section 6, I offer a few suggestions as to how massive binaries could have affected the chemical self-enrichment of globular clusters (see also de Mink et al., these proceedings).

\section{The role of binaries in population synthesis}

The influence of binaries on population number/spectral synthesis has been studied in numerous papers the last three decades (e.g., Van Bever \& Vanbeveren 2000, 2003; De Donder \& Vanbeveren 2004; Belczynski et al. 2008; and references therein). It is clear that a discussion of the evolution of binaries is essential here, especially focusing on uncertainties in all physical processes that govern binary evolution and how they affect population predictions. Let me mention stellar-wind mass loss during core hydrogen and core helium burning of massive stars, rotation, Roche-lobe overflow and mass and angularmomentum accretion in Case $\mathrm{A}$ and Case $\mathrm{Br}$ binaries, common-envelope evolution in Case $\mathrm{Bc}$ and Case $\mathrm{C}$ binaries, the spiral-in process in binaries with an extreme mass ratio, the distribution of kick velocities of a compact SN remnant and last but not least, the evolution of binary mergers. I discussed some of these uncertainties in a recent review (Vanbeveren 2009). Below, I list a number of facts resulting from all of these studies (a personal and, therefore, probably a somewhat subjective selection).

- Many (most) of the massive and intermediate-mass stars in clusters are binary members (Kouwenhoven 2006; Mason et al. 2009), but the binary frequency may vary from cluster to cluster, possibly depending on the cluster's stellar density. About half of these binaries are interacting binaries (with orbital periods less than 10 years). Note that the binary frequency among solar-type stars may be lower (Zinnecker \& Yorke 2007).

- During binary evolution, both components may merge and form a single star. When the primary of a massive binary explodes, due to the asymmetry of the SN explosion most binaries are disrupted. Due to stellar dynamics in dense clusters, binaries may be disrupted. Although binaries may also be formed due to $N$-body processes in dense clusters, the net result of all this is that the presently observed binary frequency (at least in the massive and intermediate-mass range) is lower than that at birth. This also means that a fraction of the observed single stars may have had a binary evolutionary past. To illustrate this, the single star $\zeta$ Pup in the solar neighborhood is a runaway star, which means that it most probably has had a binary history. In the past, $\zeta$ Pup has frequently been considered a prototype massive single star, but it is probably not a typical massive single star at all.

- About $10 \%$ of O-type stars are runaways (defined as stars with a peculiar space velocity $\geqslant 30 \mathrm{~km} \mathrm{~s}^{-1}$; Gies 1987). We know of two processes that can produce such stars, close encounters in dense clusters of a binary and another object (either a single star or another binary; Lada et al. 1984) and a SN explosion in massive close binaries (Blaauw 
1961). Interestingly, in the close-encounter scenario, many runaway stars are expected to be mergers of at least two stars (see, for example, the close-encounter scenario for $\zeta$ Pup in Vanbeveren et al. 2009). At present, there are too many unknown (cluster) parameters to determine the frequency of runaways formed through the close-encounter scenario. De Donder et al. (1997) proceeded as follows. Massive close binaries are a fact, SN explosions in these binaries are a fact and thus runaways formed through the Blaauw scenario are a fact. Using a binary population synthesis code, it is possible to predict this frequency. We concluded that $\sim 5-8 \%$ of O-type stars are expected to be Blaauw-type runaways (less than one third of them have a compact companion, either a NS or a $\mathrm{BH}$ ). In other words, we expect that $\sim 50-80 \%$ of O-type runaways are formed through a SN explosion in binaries, and this means that $20-50 \%$ of O-type runaways may be formed through the close-encounter process.

- The influence of recent stellar-wind mass-loss-rate formalisms (which account for the wind inhomogeneities, i.e., clumping) of massive core-hydrogen-burning stars (preluminous blue variables; LBVs) on massive-star evolution is very moderate and the uncertainties in these formalisms do not imply large uncertainties in massive-star population synthesis on the whole. However, the mass-loss rates during a possible LBV phase, the red-supergiant and WR phases (i.e., the hydrogen-deficient core-helium-burning phase of massive stars) are very important for stellar evolutionary prediction. The uncertainties in both the observed and the theoretically predicted rates are still a factor of 2 or more and unfortunately this uncertainty critically affects massive-star evolution and therefore also massive-star population synthesis predictions.

- The rotational-velocity distribution of O-type stars in the solar neighborhood reveals two important features. (i) A significant fraction are relatively slow rotators with an average velocity of $100 \mathrm{~km} \mathrm{~s}^{-1}$. When this is translated to an average initial velocity, one arrives at the conclusion that a significant fraction of O-type stars are born with an average velocity of $\sim 200 \mathrm{~km} \mathrm{~s}^{-1}$. The evolutionary calculations of the Geneva group reveal then that the effect of rotation on the pre-SN explosion of this group of massive stars is rather moderate, comparable to the effect of moderate convective-core overshooting (note that rotation implies rotational mixing of CNO elements up to the stellar surface, but this hardly affects the overall evolutionary behavior of massive stars). (ii) The distribution has an extended tail towards rapid rotators. However, a fraction of these rapid rotators are runaway stars, indicating that they may have had a binary past. Mokiem et al. (2006) studied 21 OB dwarfs in the Small Magellanic Cloud (SMC) and concluded that their average rotational velocity is $\sim 160-190 \mathrm{~km} \mathrm{~s}^{-1}$. Since massive dwarfs are very young stars close to the zero-age main sequence, the latter value should be representative for the average rotational velocity of massive stars at birth. Note then that this SMC value is very similar to the initial value of Galactic O stars while also in the SMC the most rapid rotators seem to be runaway stars, as in the Galaxy.

- Rotating single-star evolutionary models have difficulties to explain atmospheric CNO abundance anomalies in the observed massive-star sample (Hunter et al. 2008). However, the combination of rotation and binaries provides a much better correspondence (Langer et al. 2008). I think that in this discussion the massive binary HD 163181 deserves some special attention. It is an eclipsing binary with a period of 12 days, accompanied by a nitrogen-enriched BN0.5Ia primary. Hutchings (1975) derived masses for the components of 13 and $22 \mathrm{M}_{\odot}$ (for the primary and secondary, respectively). The primary is $1.5-2 \mathrm{mag}$ brighter than the secondary, which indicates that it is a core-helium-burning star that lost most of its hydrogen-rich layers through Roche-lobe overflow. This binary is therefore an illustration of the process where the atmospheric $\mathrm{N}$ enhancement is caused by binary-type mass loss rather than rotational mixing. 
- The observed overluminosity of the optical components of some of the standard massive X-ray binaries can be explained as caused by rotational mixing in spun-up mass gainers of massive binaries (Vanbeveren \& De Loore 1994).

- The effect of tides in short-period binaries on the rotation of massive binary components in low-metallicity regions (where stellar-wind mass loss is small) explains, in a straightforward way, long $\gamma$-ray bursts (Detmers et al. 2008).

- Rapidly rotating stars are formed through mass transfer in binaries or through binary mergers (due to either common-envelope evolution or dynamics in dense stellar systems). This means that a cluster in which the initial population consists mainly of slow rotators, but with a significant population of binaries, will become populated with rapid rotators as a result of binary evolution or due to the interplay of binaries and cluster dynamics. At least part of the cluster Be-type star population is expected to be formed this way (Pols \& Marinus 1994; Van Bever \& Vanbeveren 1998).

- One of the hot topics in stellar evolution research is the formation and evolution of stellar mergers. Mergers result as a consequence of canonical binary evolution (because of a nonconservative Roche-lobe-overflow process and/or common-envelope evolution) or of close encounters in star clusters where (in most cases) at least one of the players is a binary. It may therefore be expected that mergers are rapid rotators. Below, I list six different kinds of mergers which have been studied in literature, but one may think of more combinations. (i) The merger of two main-sequence (MS) stars. Smoothed-particle hydrodynamics simulations reveal that, during the merger process, large-scale mixing occurs (this means that in a cluster they will show up as blue stragglers) and mass is lost. As an example, Suzuki et al. (2007) calculated the merging of two massive MS stars (an 88+88 and an $88+28 \mathrm{M}_{\odot}$ merger). After the merger, the new star is largely homogenized (which means that this star shows the products of CNO burning in its atmosphere) while during the merger, $\sim 10 \mathrm{M}_{\odot}$ is lost. It is tempting to link these results to the $\eta$ Car event in the $19^{\text {th }}$ century. (ii) The merger of a WR and a MS star. No detailed models have been calculated but it can be expected that the resulting star may be quite spectacular. (iii) The merger of a NS (BH?) with a MS star (a Thorne-Zytkow object; Thorne \& Zytkow 1977). Calculations by Canon et al. (1992) indicate that such objects may show up as red supergiants. Their subsequent evolution is uncertain. (iv) The merger of a white dwarf (WD) and a MS star. Population synthesis of intermediate-mass binaries reveals that many WD+MS binaries merge during the common-envelope phase when the MS star fills its Roche lobe (De Donder \& Vanbeveren 2004). The subsequent evolution of these mergers is also uncertain, but I guess that it will be a rapid rotator and may show up as a Be star. (v) Double-neutron-star mergers, rapid rotators and a favorite model for short $\gamma$-ray bursts and possible sites of r-process element production and ejection (Dessart et al. 2009; see also Section 5). (vi) The merger of two WDs, a valuable model to explain SNe Ia if one accounts properly for the effects of rotation during the merger (Piersanti et al. 2003; see also Section 4).

- Accounting for the previous six points, the following statement is worth considering. "The effect of rotation is important for the evolution of some massive stars but perhaps mainly in the framework of binaries or in the framework of binaries in combination with stellar dynamics in dense clusters."

- Binaries are an essential ingredient in population number/spectral synthesis. Examples include the evolution of massive-star spectral features in starburst galaxies (Van Bever \& Vanbeveren 1998, 2003; Belkus et al. 2003; Brinchmann et al. 2008), the ultraviolet upturn in elliptical galaxies (Han et al. 2007), the X-ray binary population in galaxies (Van Bever \& Vanbeveren 2000), the population of double pulsars (De Donder \& Vanbeveren 1998, 2003; Belczynski et al. 2002), the population of carbon-enhanced 
metal-poor stars (Pols et al. 2009), the population of short $\gamma$-ray bursts (i.e., merging of double-neutron-star binaries; De Donder \& Vanbeveren 1998; O'Shaughnessy et al. 2009); the population of long $\gamma$-ray bursts (Detmers et al. 2008) and, last but not least, the SNe Ia (see Section 4) which are responsible for some $70 \%$ of all iron in the Universe.

- The discovery of the double pulsar J0737-3039 and recent population synthesis models of massive binaries have reopened the discussion on the origin of r-process elements (De Donder \& Vanbeveren 2003; Section 5).

- Massive Population III binaries, where the primary is a very massive star that ends its life in a pair-instability SN, may be important sites of primary nitrogen (Vanbeveren \& De Donder 2006).

- Most of the theoretical models that aim at explaining the chemical evolution of galaxies intrinsically assume that all stars are single (e.g., only single-star yields are used). However, most of massive stars are born as binary components and De Donder \& Vanbeveren (2004) showed that the integrated chemical yields of a population of massive binaries differs by a factor of 2-3 from the integrated chemical yields of a population of massive single stars. I think that it is time to include binaries in all Galactic chemical codes.

\section{The ultraluminous X-ray source in the young, dense cluster MGG11}

Ultraluminous X-ray sources (ULXs) are point sources with X-ray luminosities of up to $10^{42} \mathrm{erg} \mathrm{s}^{-1}$. MGG 11 is a young, dense star cluster of solar metallicity $\sim 200 \mathrm{pc}$ from the center of the starburst galaxy M82 (McCrady et al. 2003). A ULX is associated with the cluster. If the X-rays are due to Eddington-limited mass accretion onto a $\mathrm{BH}$, it is straightforward to show that the mass of the $\mathrm{BH}$ has to be at least $1000 \mathrm{M}_{\odot}$. However, how to form a star with solar metallicity and a mass greater than $1000 \mathrm{M}_{\odot}$ ? Mass segregation in a dense, young cluster associated with core collapse and the formation of a runaway stellar-collision process was promoted by Portegies Zwart et al. (2004). Note that the latter authors mainly addressed the dynamical evolution of a dense cluster, but the evolution of the very massive stellar-collision product was poorly described.

The evolution of very massive stars has been studied in detail by Belkus et al. (2007). It was concluded that stellar-wind mass loss during core hydrogen and core helium burning is very important. A convenient evolutionary recipe for such very massive stars was presented, which can easily be implemented in an $N$-body dynamical code. Our $N$-body code, which includes this recipe, has been described in Belkus (2008) and Vanbeveren et al. (2009), and was applied to simulate the evolution of MGG 11. Our main conclusion was that stellar-wind mass loss of massive and very massive stars does not prevent occurrence of a runaway collision event and the formation of a very massive star in a cluster like MGG 11. However, after this event stellar-wind mass loss during the remaining corehydrogen-burning phase is large enough to reduce the mass again and the formation of a $\mathrm{BH}$ with a mass greater than $\sim 75 \mathrm{M}_{\odot}$ is rather unlikely.

These calculations and conclusion depend critically on the adopted stellar-wind massloss formalism for very massive stars. We used a formalism proposed by Kudritzki (2002) and this requires some discussion. Kudritzki (2002) published rates for stars with luminosities up to $\log L / \mathrm{L}_{\odot}=7$, but we extrapolated his formalism for stars with mass greater than $1000 \mathrm{M}_{\odot}$, corresponding to $\log L / \mathrm{L}_{\odot} \geqslant 7.5$. Furthermore, the Kudritzki results are calculated using the Munich stellar wind code from 2000 when the line force had not yet been calculated consistently with the line blocking/blanketing NLTE (nonlocal thermal equilibrium) computations (Pauldrach 2009, priv. comm.). Using the new, more consistent version of the Munich code (for a description see Pauldrach et al. 2003) 


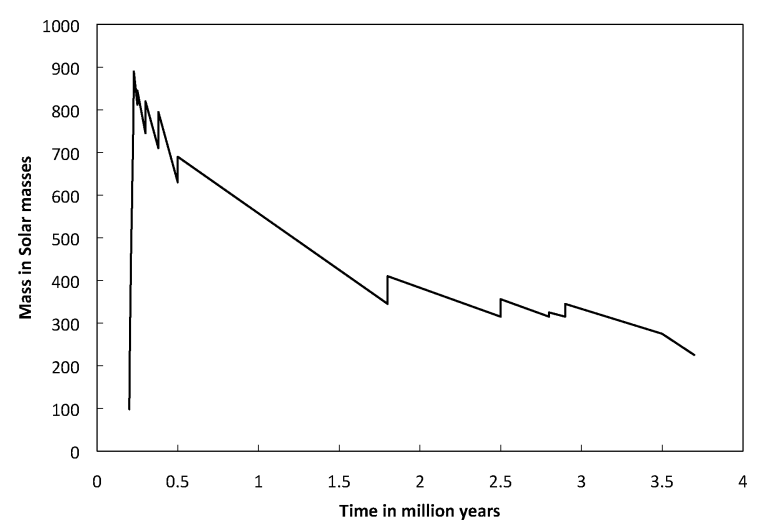

Figure 1. Mass evolution of the runaway merger in the cluster MGG 11 predicted by an $N$-body code that self-consistently includes stellar evolution of massive and very massive stars, using the stellar-wind mass formalism of Pauldrach \& Vanbeveren (in prep.).

stellar-wind mass-loss rates were determined for very massive stars with masses up to $3000 \mathrm{M}_{\odot}$ using the properties of these stars predicted by evolution (Pauldrach \& Vanbeveren, in prep.). A main result is that the new mass-loss rates are significantly smaller than the Kudritzki values. To illustrate this, for a star with $T_{\text {eff }}=50000 \mathrm{~K}$, $M / \mathrm{M}_{\odot}=250$ and $\log L / \mathrm{L}_{\odot}=6.9$, the old mass-loss rate was $2.5 \times 10^{-4} \mathrm{M}_{\odot} \mathrm{yr}^{-1}$, while the new value is $5.2 \times 10^{-5} \mathrm{M}_{\odot} \mathrm{yr}^{-1}$, or about a factor of five smaller.

We implemented the new rates in our $N$-body code (note that our code consistently combines the effects of dynamics and stellar evolution of very massive stars using a very efficient evolutionary algorithm) and we recalculated the dynamical evolution of MGG 11 using the same initial cluster conditions as in Vanbeveren et al. (2009). During core helium burning (the WR phase) of the very massive stars, we used the same WR wind formalism as in Vanbeveren et al. (2009). The new mass evolution of the runaway merger is illustrated in Figure 1. We notice a similar behavior as in the old simulations, e.g., rapid mass growth that is not prevented by stellar-wind mass loss and significant mass loss after the main collision event but, as expected, the final mass is significantly higher. The formation of an intermediate-mass BH in MGG 11 with a mass between 200 and 300 $\mathrm{M}_{\odot}$ cannot be excluded. On the question of what the maximum mass is of the $\mathrm{BH}$ formed dynamically in a cluster like MGG 11, the final answer, my friend, is clearly blowing in the stellar-wind mass loss of very massive stars.

\section{The delay-time distribution of $\mathrm{SNe}$ Ia}

It is generally believed that SNe Ia are thermonuclear explosions of WDs that exceed their Chandrasekhar limit. Two main scenarios explain how this can happen. Both involve the evolution of interacting intermediate-mass binaries, i.e., the single-degenerate (SD) and double-degenerate (DD) scenarios. In the former, the WD has a MS or a red giant companion that is filling its Roche lobe. Hydrogen-rich matter is transferred towards the WD and this pushes the WD mass over the Chandrasekhar limit. The DD model involves the formation of a double CO WD binary. Due to gravitational-wave radiation, both WDs spiral in and merge. When the mass of the merger reaches $1.4 \mathrm{M}_{\odot}$, a SN Ia may happen (Webbink 1984). It has been argued that the merger of two WDs will lead to the formation of a NS and a SN Ia will not happen (e.g., Saio and Nomoto 1998; and references therein). However, these counterarguments do not account for the consequences of rotation and 




Figure 2. Delay-time distributions (DTDs) for $\beta=1$ for the double-degenerate (DD; solid black) and single-degenerate (SD; dotted grey) scenarios as well as DTD for $\beta=0$ for the DD (dashed black) scenario. (Observational data points from Totani et al. 2008.)

angular-momentum transport during the merger. This effect was investigated by Piersanti et al. (2003; see also more recent papers published by this group) and they showed that the rotating-DD model naturally produces a hydrogenless SN Ia.

To solve the question as to which of the two scenarios is the dominant contributor to the SN Ia rate, population synthesis of starburst regions may be very useful and we focus on the delay-time distribution (DTD) of SNe Ia. The DTD is defined as the number of SN Ia events as a function of time in a starburst. By observing elliptical galaxies, which are for this purpose equivalent to starburst galaxies, at similar metallicity but at different redshifts, one can construct an observational DTD. This can then be compared to DTDs for starburst galaxies predicted by population synthesis. Such studies have been performed by Yungelson \& Livio (2000; only DD models), Han \& Podsiadlowski (2004; only SD models) and Ruiter et al. (2008; both SD and DD models). We recently performed a similar study (Mennekens et al. 2009) and I summarize some of the results here.

The theoretical DTDs are calculated using an updated version of the population code of De Donder \& Vanbeveren (2003) and we compare with the observational DTD of Totani et al. (2008). We obviously account for the common-envelope process in binary evolution, but we also focus on the evolution of Case A and Case Br binaries that evolve through a canonical Roche-lobe overflow (RLOF) process, mass transfer and mass accretion (as observed in Algol systems). Binary population synthesis results depend on many parameters and after a detailed parameter study we conclude that double WD progenitors experience two RLOF phases. The RLOF may result in the formation of a common envelope. However, our population predictions reveal that the first RLOF of most DD SN Ia progenitors is a canonical RLOF with mass transfer and mass accretion (the second RLOF when the original primary is already a WD is obviously a common-envelope process).

An important consequence of this conclusion is that any analytical formalism aimed at describing the DTD of DDs that is based on the assumption that the progenitors went through two common-envelope phases is wrong.

Figure 2 shows the DTD prediction of the DD model if the first RLOF of the progenitor binaries is assumed to be conservative (all mass lost by the loser is accreted by the 
gainer) and nonconservative (all mass lost by the loser leaves the system through the second Lagrangian point). Comparison with the observed DTD allows us to conclude that only a population model where the first RLOF is (quasi-)conservative results in a reasonable match with observations. Note that this conclusion corresponds with the situation when population synthesis predictions of Algol binaries is compared with the observed properties of Algol binaries in the solar neighborhood.

In Figure 2 we also show the DTD of the SD model using the SD progenitors of Hachisu et al. (2008). In this paper, these progenitors are identified as contours for different WD masses as a function of binary period and companion mass. A SD SN Ia is assumed to result if the evolutionary track of the progenitor system traverses this contour. As can be noticed, especially the late-time behavior of the DTD poorly matches the observations. The predicted DTD with SD SN Ia progenitors only does not reproduce the late time behavior of the observed DTD. Using DD SN Ia progenitors (eventually in combination with SD progenitors) and (quasi-)conservative RLOF in Case A and Case $\mathrm{Br}$ intermediate-mass binaries results in the best match.

\section{The delay-time distribution of NS+NS and NS+BH mergers}

It is generally accepted that the rapid neutron-capture process ( $r$-process) is responsible for the existence of the heaviest elements in the Universe. There are two sites where the physical conditions are such that the r-process can happen, i.e., in SN explosions of massive stars and in binary-neutron-star mergers (NS+NS and possibly also NS+BH) (Qian \& Woosley 1996; Rosswog et al. 2001; Dessart et al. 2009).

One of the major arguments against the binary-neutron-star merger was published by Matthews et al. (1992). He considered the observed periods and eccentricities of the binary neutron stars known at that time and calculated the expected merger-rate timescale using the theory of gravitational-wave radiation. He implemented this in a Galactic evolutionary model and when a comparison was made between the merger rate and the observed Galactic evolution of the r-process element europium, he concluded that compared to Eu double-compact-star mergers appear too late (see the dashed line in Figure 3) and thus, neutron-star-binary mergers cannot be major r-process production sites. However, the discovery of the short-period (2.4 hr) double pulsar J0737-3039 (Lyne et al.

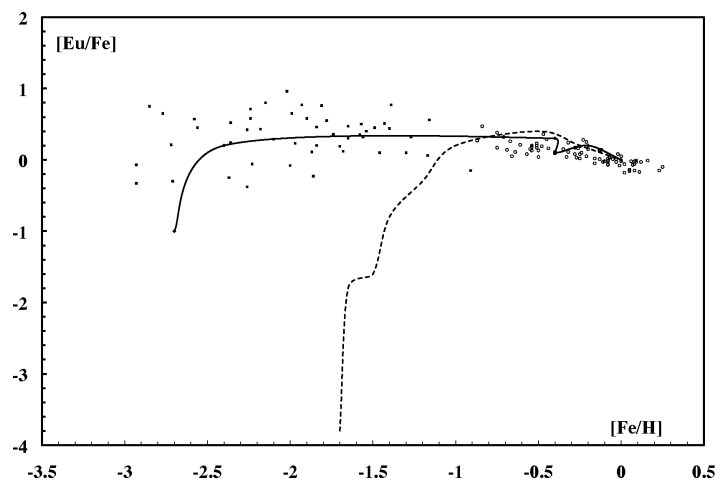

Figure 3. Temporal evolution of the predicted double-neutron-star merger rate in the solar neighborhood. The dotted curve corresponds to the prediction of Matthews (1992), while the solid line is that of De Donder \& Vanbeveren (2003, 2004). The observations are from various sources discussed in those two papers. 


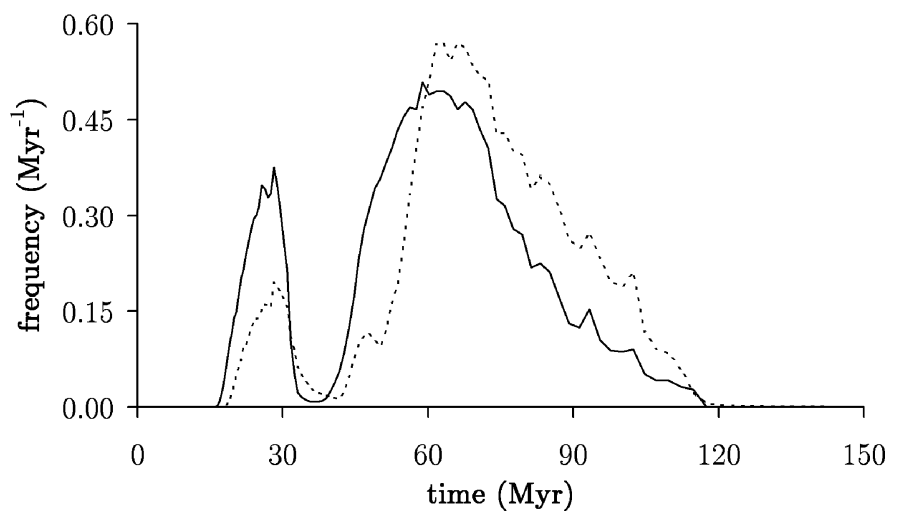

Figure 4. Delay-time distributions of double-neutron-star mergers (DTDmergers) using standard population synthesis parameters (see text). The solid and dotted curves correspond to $Z=0.02$ and 0.002 , respectively.

2004) reopened the discussion. Moreover, since 1992 our physics view of the evolution of binaries has improved significantly. Ivanova et al. (2003) and Dewi \& Pols (2003) studied the Case BB evolution of binaries with a hydrogen-deficient helium-burning component and a normal MS companion. They proposed a scenario leading to the formation of ultracompact double-neutron-star binaries which can merge on a 1 million year timescale. De Donder \& Vanbeveren $(2003,2004)$ included this scenario in a binary population synthesis model and calculated the DTD of these mergers (DTDmerger), defined as the evolution of double-neutron-star mergers in a starburst. The various evolutionary phases of massive binaries prior to double-neutron-star binary formation depend on a number of uncertain parameters and the DTDmerger also depends on these parameters. A typical distribution is shown in Figure 4 for $Z=0.02$ and 0.002 (for a Salpeter-type initial mass function, flat binary mass-ratio distribution, flat binary period distribution in the log, common-envelope evolution efficiency $\alpha=1$, mass-transfer efficiency during the canonical RLOF phase in Case Br binaries $\beta=1$ and our preferred kick-velocity distribution during the SN explosion, with an average $v_{\text {kick }}=450 \mathrm{~km} \mathrm{~s}^{-1}$ ). As can be seen, doubleneutron-star mergers appear very early. De Donder \& Vanbeveren included this model in a Galactic chemical code and Figure 3 shows that the temporal evolution of the average merger rate now follows the observed evolution of $\mathrm{Eu}$ in the solar neighborhood.

\section{The role of massive binaries in the self-enrichment of globular clusters}

It is generally accepted that a large fraction of the low-mass stars GCs are formed from material that was enriched with hydrogen-burning products produced in more massive stars: the self-enrichment process in GCs.

The first self-enrichment scenario that was proposed in the literature suggested that the enrichment was due to low-metallicity $(Z)$ intermediate-mass stars (Cottrell \& Da Costa 1981). Detailed intermediate-mass evolutionary calculations manage to explain the observed abundance patterns in GCs but fine tuning of the evolutionary processes is required, especially of those that operate during the AGB phase (e.g., Decressin et al. 2009; Ventura \& D'Antona 2009). Note that if the GC initially had an intermediate-mass binary population similar to the one in other aggregates (like in the association Sco OB2; Kouwenhoven 2006), and if the DD scenario (Webbink 1984) is responsible for the SN Ia events, population synthesis reveals that many SNe Ia should have occured in the 
past. To illustrate this, using the Brussels population code, a starburst simulation of $10^{6}$ $\mathrm{M}_{\odot}$ in stars with initial masses $\geqslant 0.8 \mathrm{M}_{\odot}$ and where $30 \%$ of the intermediate-mass stars are primaries of binaries with an orbital period $\leqslant 10$ years, predicts $10000-15000$ SN Ia events. To avoid a conflict with the observed $Z$ of GCs, it is clear that most of the matter ejected during the SNe Ia must have left the cluster. Interestingly, if the SD scenario applies we do not expect many SNe Ia at all. The reason for this is that the number of SNe Ia predicted by the SD scenario strongly depends on $Z$, and low $Z$ implies very few SNe Ia (Hachisu et al. 2008).

A second self-enrichment scenario is based on the assumption that prior to the formation of the low-mass stars, a population of low- $Z$ massive stars was present. This model may work if a process is available to remove hydrogen-burning products at low velocity (below the escape velocity of the cluster) from the low- $Z$ massive stars. Decressin et al. (2007) propose the Winds of Fast Rotating Massive Stars scenario. For this scenario to work, fast means really very fast (equatorial velocities of order 800$1000 \mathrm{~km} \mathrm{~s}^{-1}$ ) and it remains to be demonstrated whether such high average values are realistic in low-metallicity regions. However, if a massive star is a binary member, it may lose its CNO-processed layers naturally through the RLOF/common-envelope/spiral-in process and fast rotation is not needed (remember that most of the massive stars in our Galaxy are observed as members of binaries; Section 2). Therefore, I propose the RLOF/common-envelope/spiral-in Mass Loss in Massive Binaries scenario.

The DTD of merging double-neutron-star binaries was discussed in Section 5 and it was concluded that the Galactic temporal evolution of these mergers follows the observed temporal evolution of $\mathrm{Eu}$ in the solar neighborhood, which may be an indication that they are production sites of r-process elements that cannot be neglected. I suggest that these binaries may also be important sites of r-process self-enrichment of GCs.

\section{References}

Belczynski, K., Kalogera, V., Rasio, F. A., Taam, R. E., Zezas, A., Bulik, T., Maccarone, T. J., \& Ivanova, N. 2008, ApJS, 174, 223

Belczynski, K., Bulik, T., \& Kalogera, V. 2002, ApJ, 571, 147

Belkus, H. 2008, PhD Thesis, Vrije Universiteit, Brussels

Belkus, H., Van Bever, J., \& Vanbeveren, D. 2007, ApJ, 659, 1576

Belkus, H., Van Bever, J., Vanbeveren, D., \& Van Rensbergen, W. 2003, AछA, 400, 429

Blaauw, A. 1967, Bull. Astr. Inst. Netherlands, 15, 265

Brinchmann, J., Kunth, D., \& Durret, F. 2008, A\&A, 485, 657

Canon, R., Eggleton, P. P., Zytkow, A. N., \& Podsiadlowski, P. 1992, ApJ, 386, 206

Cottrell, P. L. \& Da Costa, G. S. 1981, ApJ, 245, 79

De Donder, E. \& Vanbeveren, D. 2003, NewA, 9, 1

De Donder, E. \& Vanbeveren, D. 2004, NewAR, 48, 861

De Donder, E. \& Vanbeveren, D. 1998, A\& $A$, 333, 557

De Donder, E. Vanbeveren, D., \& Van Bever, J. 1997, A\&A, 318, 812

Decressin, T., Charbonnel, C., Siess, L., Palacios, A., Meynet, G., \& Georgy, C. 2009, A\&̊A, 505,727

Decressin, T., Meynet, G., Charbonnel, C., Prantzos, N., \& Ekström, S. 2007, A\&SA, 464, 1029

Dessart, L., Ott, C.D., Burrows, A., Rosswog, S., \& Livne, E. 2009, ApJ, 690, 1681

Detmers, R. G., Langer, N., Podsiadlowski, P., \& Izzard, R. G. 2008, A\& $A, 484,831$

Dewi, J. D. M. \& Pols, O. R. 2003, MNRAS, 344, 629

Gies, D. R. 1987, ApJS, 64, 545

Hachisu, I., Kato, M., \& Nomoto, K. 2008, ApJ, 679, 1390

Han, Z. \& Podsiadlowski, P. 2004, MNRAS, 350, 1301

Han, Z., Podsiadlowski, P., \& Lynas-Gray, A. E. 2007, MNRAS, 380, 1098 
Hunter, I., Lennon, D. J., Dufton, P. L., Trundle, C., Simón-Díaz, S., Smartt, S. J., Ryans, R. S. I., \& Evans, C. J. 2008, A\&3A, 479, 541

Hutchings, J. B. 1975, ApJ, 200, 122

Ivanova, N., Belczynski, K., Kalogera, V., Rasio, F. A., \& Taam, R. E. 2003, ApJ, 592, 475

Kouwenhoven, M. B. N. 2006, PhD Thesis, University of Amsterdam

Kudritzki, R. P. 2002, ApJ, 577, 389

Lada, C. J., Margulis, M., \& Dearborn, D. 1984, ApJ, 285, 141

Langer, N., Cantiello, M., Yoon, S.-C., Hunter, I., Brott, I., Lennon, D., de Mink, S., \& Verheijdt, M. 2008, Proc. IAU Symp. No. 250, 167

Lyne, A. G., et al. 2004, Science, 303, 1153

Mason, B. D., Hartkopf, W. I., Gies, D. R., Henry, T. J., \& Helsel, J. W. 2009, AJ, 137, 3358

Matthews, G. J., Bazan, G., \& Cowan, J. J. 1992, ApJ, 391, 719

McCrady, N., Gilbert, A. M., \& Graham, J. R. 2003, ApJ., 596, 240

Mennekens, N., Vanbeveren, D., De Greve, J. P., \& De Donder, E. 2009, A\&\&A, submitted

Mokiem, M. R., et al. 2006, A\& A 456, 1131

O'Shaughnessy, R., Kalogera, V., \& Belczynski, K. 2009, ApJ, submitted (arXiv:0908.3635)

Piersanti, L., Gagliardi, S., Iben Jr., I., \& Tornambe, A. 2003, ApJ, 583, 885

Pols, O. R., Izzard, R. G., Lugaro, M., \& de Mink, S. E. 2009, Proc. IAU Symp. No. 252, 383

Pols, O. R. \& Marinus, M. 1994, A\& $A, 288,475$

Portegies Zwart, S.F., Baumgardt, H., Hut, P., Makino, J., \& McMillan, S. L. W. 2004, Nature, 428,724

Qian, Y.-Z. \& Woosley, S. E. 1996, ApJ, 471, 331

Rosswog, S., Freiburghaus, C., Thielemann, F.-K., Davies, M. B. 2001, in: J. C. Wheeler \& H. Martel, 20th Texas Symp. on relativistic astrophysics, AIP Conf. Proc. No. 586, p. 343

Ruiter, A., Belczynski, K., \& Fryer, C. 2009, ApJ, 699, 2026

Saio, H. \& Nomoto, K. 1998, ApJ, 500, 388

Suzuki, T. K., Nakasato, N., Baumgardt, H., Ibukiyama, A., Makino, J., \& Ebisuzaki, T. 2007, ApJ, 668, 435

Thorne, K. S. \& Zytkow, A. N. 1977, ApJ, 212, 832

Totani, T., Morokuma, T., Oda, T., Doi, M., \& Yasuda, N. 2008, PASJ, 60, 1327

Van Bever, J. \& Vanbeveren, D. 1998, A\& $A$, 334, 21

Van Bever, J. \& Vanbeveren, D. 2000, $A \mathscr{E} A$ A, 358, 462

Van Bever, J. \& Vanbeveren, D. 2003, $A \mathscr{E} A$, 400, 63

Vanbeveren, D. 2007, in: N. St-Louis \& A. F. J. Moffat, Massive Stars in Interacting Binaries, ASP Conf. Ser., 367, p. 13, San Francisco: Astron. Soc. Pac.

Vanbeveren, D. 2009, NewAR, 53, 27

Vanbeveren, D., Belkus, H., Van Bever, J., \& Mennekens, N. 2009, in: E. Pérez, R. de Grijs \& R. M. González Delgada, Young massive star clusters - Initial conditions and environments, ApSS, 324, 271

Vanbeveren, D. \& De Donder, E. 2006, NewA, 12, 95

Vanbeveren, D. \& De Loore, C. 1994, A\& A, 290, 129

Ventura, P. \& D'Antona, F. 2009, A\& A, 499, 835

Webbink, R. 1984, ApJ, 277, 355

Yungelson, L. \& Livio, M. 2000, ApJ, 528, 108

Zinnecker, H. \& Yorke, H. W. 2007, ARAESA, 45, 481 